\title{
Effect of meaningfulness on judgments of computability
}

\author{
DAVID E. KIERAS and JAMES G. GREENO \\ The University of Michigan, Ann Arbor, Michigan 48104
}

\begin{abstract}
After memorizing a set of four equations, subjects were given problem frames specifying an unknown variable and two, three, or four given variables. The task on each item was to judge whether the presented variables corresponded to a problem that could be computed. (No values were given and no computation was carried out.) The main finding was a strong interaction: With formulas consisting of uninterpreted letters, judgments of uncomputable items were much slower than judgments of computable items; however, the difference between positive and negative items was much smaller when formulas consisted of meaningful concepts. Attempts to remove the interaction by presenting the nonsense formulas as an integrated network or by using a format allowing easy chunking of the variables in each formula did not succeed. At this point it appears most promising to investigate effects of meaningfulness on basic component processes, such as decisions about whether pairs of items come from the same formula.
\end{abstract}

Prople retrieve information from memory in order to answer questions or to solve problems. Performance in a task requiring retrieval depends (a) on the information that is stored in memory, including its structure, and (b) on the process used by subject in retrieving information and combining elements of information so as to find an answer or a way of solving the problem.

We have studied performance in a question-answering task where the information used consists of a small network of connected concepts, determined by the experimenter. The structure of stored information thus being well defined, inferences about retrieval and combination of information should be less ambiguous; that is, there should be less uncertainty whether experimental effects are the result of retrieval and transformation processes or of properties of cognitive structure.

The knowledge base for the task was a set of four simple equations, memorized by the subject. The subject then answered a long series of questions, each involving the "computability" of a problem. In each question, a variable from the equations was designated as "unknown" and two, three, or four "given" variables were listed. No values were presented for the variables, and the subject carried out no computation. The question answered by the subject was whether the value of the unknown variable could be calculated if values of the given variables were known. The time taken by the subject in making correct responses was the dependent variable.

The task used in the present studies relates closely to the question-answering task studied by Collins and Quillian (1969, 1970), Conrad (1972), Meyer (1970), and others, in which the subject evaluates sentences such as "All cars are vehicles" or "Lions have fenders," as being

This research was supported by National Science Foundation Grant No. GB-31045.

The first author is now at Carnegie-Mellon University. true or false. The task also shares features of a retrieval problem studied by Hayes (1966), in which the subject is taught a series of connections between the names of spies, with the connections referring to the pairs of spies who can give each other messages, and the subject is given the task of finding a way to get a message between two spies specified by the experimenter. In all of the tasks, the subject must respond on the basis of connections beiween concepts in the network that constitutes the knowledge base. In our task, as in the studies of retrieval from general semantic memory, the subject responds "yes" if the knowledge base contains appropriate connections among the concepts presented. Like the spy problems, and unlike the studies of semantic memory, our task uses a small, well defined network of concepts rather than the subject's general knowledge. The correct response in our task depends on the existence of a set of connections in the network, rather than the specific relations involved. Thus, if $A$ can be computed from $B$ and $C$, then $B$ can be computed from $A$ and $C$. This contrasts with studies of general semantic memory where the kind of connection matters; for example, "All cars are vehicles" is true, but "All vehicles are cars" is false. Finally, our task uses relations that are not binary. Each of the formulas in our knowledge base had one variable connected to two others, a trinary relation, whereas other studies have involved binary relations, such as a connection between class names and properties (canaries are yellow), or subset and superset relations (canaries are birds), or between pairs of spies who communicate with each other.

As it has turned out, our studies have provided information mainly about the effect on retrieval and processing of having concepts in the knowledge base that are also in subject's general semantic memory. One set of equations, learned by some subjects, had variables corresponding to familiar concepts, such as distance, 
Table 1

Formulas Used in Experiment I

\begin{tabular}{ll} 
Meaningful & Nonsense \\
\hline Driving time $=$ arrival time - leaving time & $\mathrm{V}=\mathrm{F}-\mathrm{L}$ \\
Distance $=$ driving time ${ }^{*}$ average speed & $\mathrm{D}=\mathrm{V} * \mathrm{~A}$ \\
Gas mileage $=$ distance/gas used & $\mathrm{M}=\mathrm{D} / \mathrm{G}$ \\
Total time $=$ driving time + preparation time & $\mathrm{T}=\mathrm{V}+\mathrm{P}$ \\
\hline
\end{tabular}

driving time, speed, and gasoline mileage. Another set of equations, learned by a separate group of subjects, had variables designated by single letters without any obvious meaning. The familiar concepts gave meaningful formulas, in the sense that they can be interpreted in relation to subjects' general knowledge. The formulas made up of uninterpreted letters are nonsense, in the usual sense that they do not relate in any obvious ways to general knowledge of the subjects.

\section{EXPERIMENT I}

Our first study was exploratory, intended to determine whether the task was a reasonable one for subjects, and whether the meaningfulness of equations in the knowledge base would have any interesting effect on decision latencies.

\section{Method}

Design, Materials, and Subjects. Two groups of 10 subjects were used, one group having meaningful formulas, the other nonsense. The meaningful equations expressed valid relations among variables involved in an hypothetical highway trip, which was described by an accompanying paragraph. The nonsense equations were produced by substituting arbitrary letter variable names for the word variable names in the meaningful equations. The two sets of equations are shown in Table 1.

Matched sets of test questions were constructed from the meaningful and nonsense formulas, with the questions made from nonsense variables formed by substituting variable for variable into the questions made from meaningful variables according to the correspondence shown in Table 1. The questions were generated by a computer program according to the following rules: Only variables appearing in the equations were used. The number of given variables was either two, three, or four. All of the 36 possible positive questions (correct answer is "yes") were included, along with 36 negative questions (correct answer is "no") that were randomly generated with approximately the same distribution of the number of given variables as the positive questions. In neither positive nor negative questions were there any given variables that could be obtained from other given variables.

Subjects were University of Michigan undergraduates who gave only correct answers on a screening test requiring solution of linear equations, intended to eliminate subjects who lacked a working knowledge of simple algebra. Subjects were paid for their participation in the experiment.

Procedure. Subjects were tested individually and assigned to a materials condition by means of a coin flip. At the start of each session, the subject was given a list of the four equations and instructed to memorize them and be able to answer questions about them. The subject was left alone to study the equations for $15 \mathrm{~min}$, and then performed a distractor task of listing all the permutations of the digits, $1,2,3$, and 4 . Then the subject wrote out the four equations from memory. All subjects gave all four equations correctly in this test, although some latitude in spelling, form, and recall order was allowed.
The experimenter explained the main task to the subject, including the way in which questions were to be presented. Instructions stressed accuracy over speed by suggesting that the subject should take as much time as needed to be accurate, but no more.

A different random order of the 72 questions was generated for each subject in the group with meaningful formulas, and a matched order of corresponding questions was used for a subject with nonsense formulas. The questions were punched and interpreted on tabulating cards, with the unknown variable on the left followed by a dash, then the list of given variables. The set of cards was duplicated to give a set of 144 questions in two identical blocks of 72 for each subject. (When questioned, no subject reported noticing the duplication.)

On each trial, the experimenter simultaneously started a chronoscope and signaled the subject, who turned up and read a question card. When subject gave a yes or no answer, the experimenter stopped the chronoscope, took the card from subject, and recorded the time and subject's answer on the card. No feedback was given. Usually the experimenter was not aware of the correct answer. A short break was given between the two blocks of questions. The total session time was about $90 \mathrm{~min}$.

\section{Results}

In all the experiments to be reported, latencies for incorrect answers were excluded from analysis, which was performed on the mean latencies for each combination of number of givens and correct answer; each subject thus contributed six data points. In this first experiment, error rates were $8 \%$ for meaningful and $12 \%$ for nonsense formulas.

The main results of the experiment are in Figure 1,

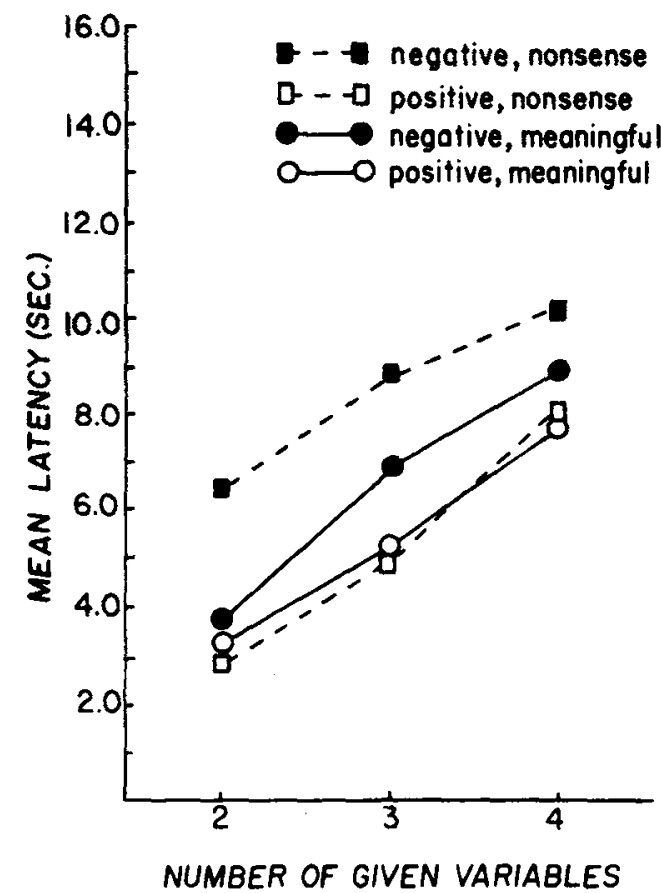

Figure 1. Mean latencies from the second block of Experiment I for positive and negative questions from meaningful and nonsense formulas as a function of number of givens. The approximate standard errors for the mean latencies, obtained according to Cochran and Cox (1957, Chap. 7) from error mean squares, are $.63 \mathrm{sec}$ for within-subject comparisons and $1.96 \mathrm{sec}$ for between-subject comparisons. 
which presents for the second block of trials the mean latency of all correct responses for questions with different numbers of given variables and different correct answers for the two groups of subjects. Analysis of variance showed that the main effect of the number of given variables was significant $[F(2,18)=40.61, p<.001]$, as was the main effect of the difference between positive and negative items $[F(1,18)=26.20, p<.001]$. The main effect of meaningfulness of formulas was not significant $[F(1,18)=1.20, p<.10]$. The same trends appeared in both blocks. However, response latency was shorter on the second block than on the first $[F(1,18)=29.20, p<.001]$ and the effect of number of givens on latericy was less in the second block than the first, as indicated by the interaction between blocks and number of givens $[F(2,36)=5.74, p<.01]$. The effect of number of givens was apparently not different between the two groups: The interaction between number of givens and meaningfulness was not significant $[\mathrm{F}(2,18)<1]$. Of greatest interest was the interaction between meaningfulness and the correct answer. The difference between positive and negative items was significantly greater in the groups with nonsense formulas than in the group with meaningful formulas $[F(1,18)=8.53, p<.01]$.

\section{Discussion}

The fact that the number of given variables had a significant effect on latency mainly indicates that the task yields reasonable and reliable results. The situation is complicated regarding negative items, but with positive items the number of given variables corresponds directly to the number of formulas that subject must retrieve from memory to form a connected path from the given variables to the unknown. In other words, more given variables correspond to a greater number of nodes that must be traversed, in the sense of Collins and Quillian (1969, 1970), and the greater the number of connections involved, the more time was required.

We do not have a strong interpretation for the finding that negative items took longer to decide than positive items, or for the approximate equivalence of this difference with questions differing in the number of given variables. There are several strategies available to subjects for making decisions about computability, and many of them require more steps for most of the negative items than for positive items of the same length. However, an exact analysis would require knowing the strategy used. There is reason to believe that different subjects use different strategies (Malin, 1973) or that different strategies are used on different problems, and latency data are not sufficient to identify strategies. On the other hand, the finding that negative items take longer than positive items is not unusual in research of this kind (e.g., Collins \& Quillian, 1972).

The interaction found between correct answer and meaningfulness similarly lacks a definite interpretation,
Table 2

Formulas Used in Experiment II

\begin{tabular}{ll}
\hline Meaningful & Nonsense \\
\hline Driving time $=$ arrival time - leaving time & $\mathrm{E}=\mathrm{B}-\mathrm{M}$ \\
Distance $=$ speed ${ }^{*}$ driving time & $\mathrm{I}=\mathrm{T} * \mathrm{E}$ \\
Distance $=$ gas mileage $^{*}$ gas used & $\mathrm{I}=\mathrm{N} * \mathrm{H}$ \\
Speed $=$ wheel size ${ }^{*}$ wheel speed & $\mathrm{T}=\mathrm{G} * \mathrm{X}$ \\
\hline
\end{tabular}

but it may show a general property of the difference between deciding positive and negative items. To decide that "no" is the correct answer, subject probably determined that the given variables were not sufficient to reach the unknown variable in the network. This process could take a long time, especially if individual variables had to be considered and all possible connections had to be checked. The finding of a smaller difference between positive and negative items for the meaningful formulas suggests the possibility that in some way subjects were able to treat the network of meaningful formulas in a more integrated way, perhaps by utilizing a kind of look-ahead in the network search or by processing on the basis of combinations of variables rather than single variables. Experiments II and III were carried out to check this possibility in more specific forms.

\section{EXPERIMENT II}

The possibility checked in this study was that meaningful formulas might be represented in memory as an interconnected network, more so than the nonsense formulas, which might be represented more as a list of relatively distinct elements. Our idea was that presentation of a network representation might be the basis for a more integrated kind of processing due to having a more integrated or wholistic representation of the knowledge base.

\section{Method}

Design, materials, and subjects. There were six groups in this experiment, with a 2 by 3 factorial design. One of the factors was the meaningfulness of the formulas memorized by subjects, varied as in Experiment I. The other factor was supplemental training given after subjects had memorized the four equations.

The equations used in this study are shown in Table 2, in both their meaningful and nonsense forms. The relationships among variables in this set of equations are somewhat more symmetrical, making possible easy replication of types of problems requiring similar sequences of steps for solution.

The three conditions of supplemental training were (1) no supplement, (2) training using a graphic network of the formulas, and (3) training using rote drill. In the network supplement, the equations were displayed to subject in a kind of "organization chart," having the central equation (Distance $=$ Speed ${ }^{*}$ Driving Time, or $I=T^{*} E$ ) at the top of the display, with the other variables "hung" from the variables in the central equation. The approximate appearance of the display of the nonsense equations is shown in Figure 2. After studying this display, subjects were trained to fill in missing portions of the chart, and answered a series of six questions repeated to a criterion of all correct. 


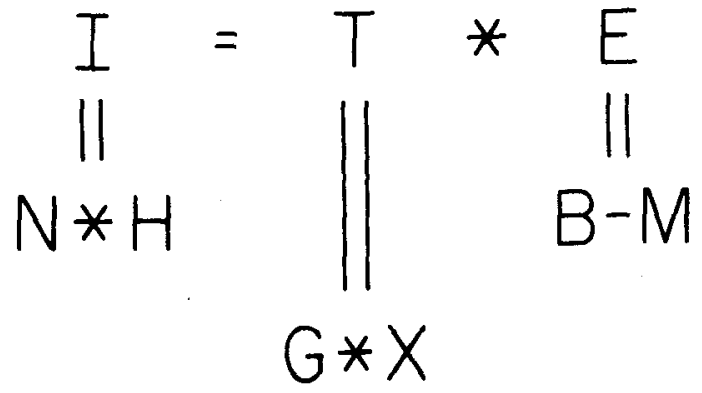

Figure 2. Graphical network display of nonsense equations in Experiment II.

The rote drill supplement was designed as a general practice control, intended to give subjects extra practice in recalling equations without encouraging them to relate the equations together. The subject answered a list of 12 questions of the form: "What equation uses the variables $B$ and $M$ ?" The subject was required to give the equation and was given feedback, and the 12 questions were repeated until all were answered correctly.

The questions selected for the main task of judging computability included all of the 47 possible positive questions that can be made with no redundant given variables, and a set of 48 negative questions chosen with a number of constraints. For example, no question was included in which the unknown was a member of only one equation unless the other unique unknown in that equation was in the list of given variables. As in Experiment I, questions based on the nonsense formulas were formed by substituting corresponding variables in the questions based on meaningful formulas, giving completely isomorphic sets of questions.

The subjects were 66 Unjersity of Michigan undergraduates who participated for pay, and were randomly assigned to groups. No screening test for algebraic knowledge was used, but subjects making more than $20 \%$ errors in the question task were replaced.

Appartus and Procedure. Materials were presented on IBM 2260 display/keyboard devices which were controlled by an IBM 1800 computer which also recorded the latency data (Kieras, 1973). Subjects responded using the keyboards during training on the formulas, and with pushbuttons during the main question task. Up to five subjects were tested in each experimental session, and typically subjects in each session were all tested in different conditions.

At the beginning of the experiment, subjects received instructions through the display/keyboard terminals, including notation, use of the keyboards, and use of the pushbuttons. Subjects who were to receive meaningful formulas then were shown a series of displays describing the hypothetical highway trip and mentioning the variables. After the four equations were displayed to the subject for as long as the subject wanted to study them, the subject was asked to type all four equations on the keyboard, one at a time. The computer required exact answers, except for character spacing. If one or more of the recalled equations was incorrect, the set of equations was displayed again, until the subject indicated being finished, and then the subject was asked again to type the four equations.

When the subject typed all four equations successfully, the training supplement (if any) was given. Following that, the subject performed a distractor task of typing in some personal information including name and student identification number, and typing the alphabet backward. At this point, the subject was required to type the four equations again, returning to the display of equations if necessary before proceeding. Finally, instructions were presented explaining the question task, followed by the question series, with subjects pacing themselves and no feedback given. On each trial, the unknown appeared in the upper left corner of the display screen and the given variables were in a vertical list on the right side of the screen. The order of variables in the list of givens and the order of presentation of the 95 questions were freshly randomized before each session. The total time for each session was about $90 \mathrm{~min}$.

\section{Results}

The main results appear in Table 3 , which shows mean latencies collapsed over number of givens for each combination of meaningfulness, similarity, and correct answer. Number of givens had a significant main effect $[F(2,108)=116, p<.001]$ and interacted with meaningfulness $[\mathrm{F}(2,108)=12.53, \mathrm{p}<.001]$ and correct answer $[F(2,108)=3.78, p<.01]$. However, these effects of number of givens are not shown in Table 3 since they are not very interesting and do not have obvious theoretical import.

The supplementary training manipulation did not affect response latency and also failed to modify any of the effect produced by the other factors. The main effect of supplement was nonsignificant $[F(2,54)=2.29$, $\mathrm{p}>.10]$ and all interactions between supplements and other factors were nonsignificant. Meaningfulness had a significant main effect $[F(1,54)=6.66, p<.025]$ as did correct answer of the questions $[F(1,54)=42.20$, $\mathrm{p}<.001]$. The interaction between meaningfulness and correct answer observed in Experiment I was also significant in this experiment $[F(1,17)=23.99$, $p<.001]$.

\section{Discussion}

The failure of the experiment to give a significant three-way interaction between meaningfulness, correct answer, and supplementary training means that the experiment failed in its main objective of identifying a manipulation that would produce processing of nonsense formulas significantly more similar to that of meaningful formulas. The feature that makes the result noteworthy is the definite lack of encouragement for the idea that representing the formulas in a network has anything to do with the effect of meaningfulness on processing. The rote drill condition was intended as a general practice control. If anything, it was more effective than the network presentation in producing a condition in which processing of nonsense formulas was similar to processing of meaningful formulas.

Table 3

Mean Latencies* in Seconds from Experiment II for Positive and Negative Questions Collapsed Over Number of Givens

\begin{tabular}{lccccc}
\hline & \multicolumn{3}{c}{ Meaningfulness } \\
\cline { 2 - 3 } \multicolumn{1}{c}{ Supple- } & \multicolumn{2}{c}{ Nonsense } & & \multicolumn{2}{c}{ Meaningful } \\
\cline { 2 - 3 } \cline { 5 - 6 } ment & Positive & Negative & & Positive & Negative \\
\hline None & 9.49 & 16.33 & & 10.41 & 10.77 \\
Rote Drill & 8.53 & 11.59 & & 7.53 & 8.12 \\
Network & 9.23 & 14.08 & & 6.99 & 7.93 \\
\hline
\end{tabular}

*Approximate standard errors for the cell means are $.75 \mathrm{sec}$ for within-subject and 1.49 sec for between-subject comparisons (Cochran \& Cox, 1957, Chapter 7). 
The idea of a network presentation was motivated by an hypothesis that meaningful formulas are represented in a more integrated overall manner. The results of this experiment suggest a different hypothesis, namely, that meaningful formulas are integrated more strongly as trigram units. If this is the case, then perhaps the difference in processing meaningful and nonsense formulas occurs because members of meaningful formulas stand out in the list of variables as "belonging together" in the same chunk, and thereby permit the subject to use more efficient retrieval strategies based on accessing large chunks with multiple cues. On this hypothesis, the nonsense equations constrain the subject to variable-by-variable processing by providing no basis for such higher level organization.

\section{EXPERIMENT III}

An implication of the idea that chunking of individual formulas accounts for the interaction between meaningfulness and correct answer is that nonsense formulas might be processed similarly to meaningful formulas if they were presented in a way that makes chunking easy. Perceptual similarity, produced by giving variables names with shared symbols, was used. Again, the question is whether an experimental manipulation, applied to the nonsense materials, would produce a condition in which processing is more similar to that of the meaningful formulas, and the indicator of that processing was taken to be the difference between times to process positive and negative questions.

\section{Method}

Design, materials, and subjects. The design was a 2 by 2 factorial, with meaningfulness of formulas as one of the factors, and the other being the formal similarity of the names of variables within formulas. We realized that the meaningful formulas used in the earlier studies had a considerable amount of formal overlap in the names of variables (for example, "driving time = arrival time - leaving time"), so we constructed a set of equations with meaningful variables that had considerably less overlap than those used previously, and another set that had a bit more overlap. The nonsense equations with formal overlap were constructed by use of subscripted variables. All the formulas used are shown in Table 4.

The items for the computability judgments were a set of 202 questions, using all of the 47 positive items twice each, and 108

Table 4

Formulas Used in Experiments III and IV

\begin{tabular}{ll}
\hline Meaningful & Nonsense \\
\hline Similar & \\
Distance $=$ driving speed $*$ driving time & $\mathrm{X} 1=\mathrm{Y} 1 * \mathrm{Z} 1$ \\
Distance $=$ gas mileage ${ }^{*}$ gas used & $\mathrm{X} 1=\mathrm{X} 2 * \mathrm{X} 3$ \\
Driving speed $=$ wheel size $*$ wheel speed & $\mathrm{Y} 1=\mathrm{Y} 2 * \mathrm{Y} 3$ \\
$\begin{array}{l}\text { Driving time }=\text { total time }- \text { stop time } \\
\text { Dissimilar }\end{array}$ & $\mathrm{Z} 1=\mathrm{Z} 2-\mathrm{Z3}$ \\
Distance $=$ speed $*$ driving time & $\mathrm{I}=\mathrm{T} * \mathrm{E}$ \\
Distance $=$ mileage $*$ gas used & $\mathrm{I}=\mathrm{N} * \mathrm{H}$ \\
Speed $=$ force $/$ weight & $\mathrm{T}=\mathrm{G} * \mathrm{X}$ \\
Driving time $=$ duration - stops & $\mathrm{E}=\mathrm{B}-\mathrm{M}$ \\
\hline
\end{tabular}

Table 5

Mean Latencies* in Seconds from Experiment III for Positive and Negative Questions Collapsed Over Number of Givens

\begin{tabular}{lccccc}
\hline & \multicolumn{4}{c}{ Meaningfulness } \\
\cline { 2 - 5 } Similarity & Positive & Negative & & Positive & Negative \\
\cline { 2 - 5 } \cline { 5 - 6 } Similar & 5.06 & 7.81 & & 7.30 & 9.93 \\
Dissimilar & 6.59 & 12.24 & & 8.74 & 11.36 \\
\hline
\end{tabular}

*Approximate standard errors for the cell means are $.54 \mathrm{sec}$ for within-subject and $.84 \mathrm{sec}$ for between-subject comparisons (Cochran \& Cox, 1957, Chapter 7).

negative items. The negative items were selected using a rough model intended to show whether subjects were answering the questions by means of "backward" or "forward" strategies (see Malin, 1973). However, since nothing noteworthy resulted from this manipulation, it will not be reported any further, except to note that as in the previous experiments, all conditions received the same set of questions, which differed only in the variable names.

The 44 subjects were from the source as in Experiments I and II, and were randomly assigned to the four groups.

Apparatus and procedure. The experiment was conducted in the same way as Experiment II, except, of course, no supplementary training was given. Total time for each session was about $2 \mathrm{~h}$.

\section{Results}

The mean latencies collapsed across number of givens for each combination of similarity, meaningfulness, and correct answer are shown in Table 5. As before, although number of givens had a main effect $[F(2,80)=253.00$, $\mathrm{p}<.001]$ and interacted with similarity $[F(2,80)=9.32, \quad \mathrm{p}<.001]$ and correct answer $[F(2,80)=4.62, p<.025]$, these effects are not shown in Table 5, for the sake of brevity.

Correct answer had a singificant main effect $[F(1,40)=80.79, \quad p<.001]$ as did similarity $[F(1,40)=8.71, p<.01]$ but the main effect of meaningfulness was marginal $[F(1,40)=3.52$, $.05<\mathrm{p}<.10]$ as was the interaction between similarity and correct answer $[F(1,40)=3.65, .05<p<.10]$.

The key result in this study was the marginally significant three-way interaction between meaningfulness, similarity, and correct answer $[\mathrm{F}(1,40)=3.69, .05<\mathrm{p}<.10]$, which suggests that similarity caused nonsense material to be processed similarly to meaningful material. Because of the importance of this marginal interaction, the experiment was repeated with certain minor changes in equipment and procedure, and 19 subjects drawn from the same population were randomly assigned to each group. Many of the effects failed to replicate; only the significant effects resemble their counterparts in the original study. Main effects of correct answer $[F(1,72)=72.8$, $p<.001]$ and number of givens $[F(2,144)=2.97$, $\mathrm{p}<.001]$ were significant; the only significant interaction was between meaningfulness and correct answer $[F(1,72)=17.7, p<.005]$. All other 
interactions, including the marginal interaction between meaningfulness, similarity, and correct answer, failed to approach significance (all values of $F<2$ ).

\section{Discussion}

The last set of results offers no support for the hypothesis that meaningfulness and perceptual similarity produce similar effects by promoting chunking of the materials, since the interaction between meaningfulness, similarity, and correct answer failed to replicate. It is hard for us to believe that the minor changes made between the original experiment and the replication could account for the failure to replicate the interaction. Thus, if there is this interaction due to chunking, it is very weak compared to the Meaningfulness by Correct Answer interaction which has persisted in all four studies. However, the trends do seem to be present in the data; perhaps more sensitive techniques would reveal definite effects of similarity.

\section{GENERAL DISCUSSION}

The results reported in this paper are negative in the sense that our attempts to manipulate the original Meaningfulness by Correct Answer interaction observed in Experiment I all met with failure. Although the results of Experiments II and III were primarily negative, it seems to us that they are useful for theory for two reasons: (a) The original interaction between answer and meaningfulness was quite robust, appearing in different procedures and materials, and over a large number of subjects; this interaction is important for theory because it is strong evidence that processing of meaningful material differs in some substantial way from that of meaningless material. This aspect of the results is definitely positive in nature. (b) Our attempts to manipulate this important interaction were intuitively reasonable and involved variations that seem to us to be reasonably major differences between conditions. If the reader agrees with this assessment of our manipulations, he will be willing to conclude that some information has been gained by these negative results about the nature of the difference between meaningful and meaningless material. The difference obviously cannot be attributed to differences in the formal structure of the material, since in all four studies, the formal structures for the different equation sets were identical. Nonsense was not rendered more meaningful by enhancing its acquisition-time organization, in terms of either intra- or interequation relationships. Apparently, meaningfulness lies in organizational factors that are not contained in the equations themselves, but depend on subject's general knowledge.

An important feature of our results to be kept in mind is that meaningfulness did not produce a general facilitation of computability decision latencies. For positive times, meaningful material apparently led to about the same latency as nonsense in Experiment I, and greater latency in Experiment III. While negative nonsense items appeared slower than negative meaningful items in most cases, even that generalization has one exception in the data of Experiment III. The fact to be explained is not a general facilitation of computability decisions due to meaningfulness, but a smaller difference between positive and negative items when materials are meaningful than when they involve nonsense.

Although we have not been able to produce successful models for the computability task, we can sketch the dimensions of the theoretical problem and promising routes of possible explanations and further investigation. Following Atkinson and Shiffrin's (1968) distinction between permanent structural capabilities and control processes determining the use of structural capabilities, we can distinguish between the two broad categories of subprocesses involved in the computability task: (a) the basic retrieval and comparison processes used to retrieve equations from memory and test equations in working memory for the presence or absence of variables, and (b) the problem-solving strategy used by the subject to determine the sequence of retrieval and comparison operations. With only our unsupplemented latency data, it is not possible to distinguish whether the Meaningfulness by Correct Answer interaction is due to difference in basic processes or strategies for meaningful and nonsense material. However, it seems reasonable to assert that the only reason for subjects using a different problem-solving strategy would be to take advantage of a difference in the basic processes available with meaningful materials. Hence the plausible explanations for the interaction are limited to either a difference in basic processes only, or a difference in both basic processes and problem-solving strategy.

Malin's (1973) approach to the issue of different strategies may be helpful to further investigations. Malin had subjects provide verbal traces of the formulas they retrieved from memory as they constructed solutions, and she was thus able to identify general features of subjects' strategies from the protocols. Malin found a difference in choice of strategies for different materials, but the difference was related mainly to physical features of stimuli that facilitate chunking rather than to meaningfulness. With the results of Experiment IV we are inclined against explanations of the Meaningfulness by Correct Answer interaction that depend on simple chunking based on physical features. Of course, Malin's materials might not have provided enough contrast between meaningful and nonsense conditions. Furthermore, Malin used only positive questions, which suggests that her strategy-choice observations might not apply directly to our computability task.

Concerning basic retrieval and comparison processes, one component of the task required the subject to com- 
pare pairs of individual items and determine whether they are in a single formula. Sometimes the pair of items was presented in the stimulus, and sometimes one or both items to be compared were retrieved from memory. Effects of semantic meaningfulness on this comparison process could have rather large effects on the overall process of judging computability. One indication that augers for such an effect is the result obtained by Meyer and Schvaneveldt (1971), who found faster judgments that letter strings are words when the strings were associatively related words than if the strings were unrelated words. More direct evidence on the matter would come from asking subjects to judge whether a pair of items (words or letters) comes from a single formula or from different formulas. In preliminary results using this task, David King (personal communication) has observed that judgments for items from a single formula take about the same amount of time whether the materials involved meaningful concepts or nonsense letters. However, judgments that items come from different formulas appear to be faster if the materials are meaningful than if they are nonsense.

As mentioned above, in our computability judgment data, there is no general decrease in response latencies for meaningful material compared to nonsense material. Hence, the notion that meaningful material is manipulated faster than nonsense cannot be supported directly by our data; however, the notion cannot be rejected since a general facilitation of basic manipulations is confounded with differences in uninteresting properties of the materials, such as reading times. Without some means of specifying the number of basic operations required for different items, we cannot separate manipulation times from the uninteresting times. Furthermore, we think that the problem-solving strategies probably are different for the two types of material. It seems likely that our inability to specifically determine the nature of the processing difference between meaningful and nonsense material was due to our lack of a method for either controlling or inferring the problerr-solving strategies used by subjects. On the other hand, these experiments provide strong evidence that the semantic meaningfulness of task materials has important effects in problem-solving situations (cf. Greeno, 1973) and this dependence merits further investigation.

\section{REFERENCES}

Atkinson, R. C., Shiffrin, R. M. Human memory: A proposed system and its control processes. In R. W. Spence and J. T. Spence (Eds.), Advances in the psychology of learning and motivation. Vol. 2. New York: Academic Press, 1968.

Cochran, W. G., \& Cox, G. M. Experimental designs. 2nd Ed. New York: Wiley, 1957.

Collins, A. M., \& Quilian, M. R. Retrieval time from semantic memory. Journal of Verbal Learning \& Verbal Behavior $1969,8,247-249$.

Collins, A. M. \& Quillian, M. R. Does category size affect categorization time? Joumal of Verbal Learning \& Verbal Behavior, $1970,9,432-438$

Collins, A. M., \& Quillian, M. R. How to make a Ianguage user. In E. Tulving and W. Donaldson (Eds.), Organization of memory. New York: Academic Press, 1972. Pp. 310-354.

Conrad, C. Cognitive economy in semantic memory. Joumal of Experimental Psychology, 1972, 92, 149-154.

Greeno, J. G. The structure of memory and the process of solving problems. In R. Solso (Ed.), Contemporary issues in cognitive psychology: The Loyola symposium. Washington, D.C: Winston, 1973. Pp. 103-154.

Hayes, J. R. Memory, goals and problem solving. In B. Kleinmuntz (Ed.), Problem solving research, method, and theory. New York: Wiley, 1966. Pp. 149-170.

Kieras, D. A general experiment programming system for the IBM 1800. Behavior Research Methods \& Instrumentation, $1973,5,235-239$.

Meyer, D. E. On the representation and retrieval of stored semantic information. Cognitive Psychology, 1970, 1 , 242-300.

Meyer, D. E., \& Schvaneveldt, R. W. Facilitation in recognizing pairs of words: Evidence of a dependence between retrieval operations. Journal of Experimental Psychology, 1971, 90, 227-234.

\section{REFERENCE NOTE}

Malin, J. E. T. An analysis of strategies for solving certain substitution problems. Human Performance Center Technical Report No. 40, University of Michigan, April 1973.

(Received for publication January 14, 1974; revision received September 23, 1974.) 